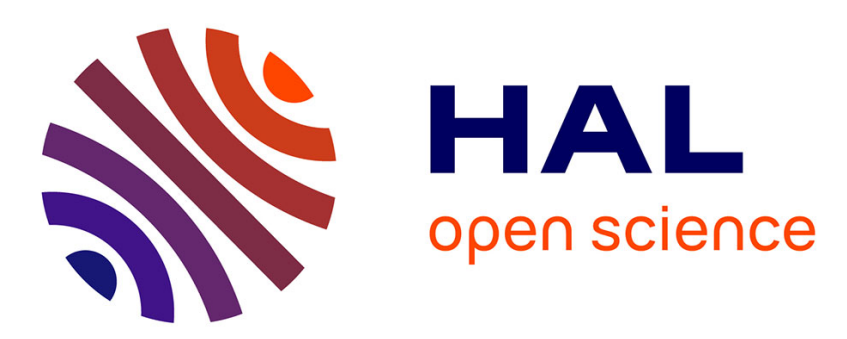

\title{
Coexistence of IEEE 802.11p and the TDMA-based AS-DTMAC Protocol
}

Fouzi Boukhalfa, Mohamed Hadded, Paul Mühlethaler, Oyunchimeg Shagdar

\section{To cite this version:}

Fouzi Boukhalfa, Mohamed Hadded, Paul Mühlethaler, Oyunchimeg Shagdar. Coexistence of IEEE 802.11p and the TDMA-based AS-DTMAC Protocol. SoftCOM 2020: International Conference on Software, Telecommunications and Computer Networks 2020, Sep 2020, Hvar / Virtual, Croatia. hal02981085

\section{HAL Id: hal-02981085 \\ https://hal.science/hal-02981085}

Submitted on 27 Oct 2020

HAL is a multi-disciplinary open access archive for the deposit and dissemination of scientific research documents, whether they are published or not. The documents may come from teaching and research institutions in France or abroad, or from public or private research centers.
L'archive ouverte pluridisciplinaire HAL, est destinée au dépôt et à la diffusion de documents scientifiques de niveau recherche, publiés ou non, émanant des établissements d'enseignement et de recherche français ou étrangers, des laboratoires publics ou privés. 


\title{
Coexistence of IEEE 802.11p and the TDMA-based AS-DTMAC Protocol
}

\author{
Fouzi Boukhalfa*, Mohamed Hadded*, Paul Muhlethaler ${ }^{\dagger}$ and Oyunchimeg Shagdar* \\ *Institute VEDECOM, 23 bis allée des Marronniers, 78000 Versailles, France \\ ${ }^{\dagger}$ EVA Team, Inria Paris, 2 Rue Simone Iff, 75012 Paris, France
}

\begin{abstract}
Advanced vehicular applications such as autonomous driving are leading to a new evolution in vehicular radio access technologies. The Task Group BD on the NextGeneration V2X (NGV) have defined IEEE 802.11bd as the new standard for the Dedicated Short Range Communications (DSRC). Notwithstanding its the promising performances in terms of high reliability, low latency and high throughput, the design must also respect certain specifications, such as coexistence, interoperability, and compatibility with the previous standard. In this article, we study how the IEEE 802.11p protocol could coexist with a TDMA-based protocol named AS-DTMAC, which has recently been proposed to control access in Vehicular Ad Hoc Networks (VANETs). We carry out several analyses to show that the two protocols can coexist by several analysis when both are operating simultaneously on the same network. We also propose a modification of AS-DTMAC to handle the situation where both IEEE 802.11p and AS-DTMAC have to send urgent packets.
\end{abstract}

Keywords - VANETs, IEEE 802.11p, TDMA, Active signaling, Coexistence, Low latency, Network simulation.

\section{INTRODUCTION AND MOTIVATION}

Vehicular Adhoc NETworks (VANETs) have attracted considerable attention both in academic communities and industrial communities [1]. VANETs allow communications between moving vehicles with the aim of establishing data exchange and collaboration between them for which different safety, commercial and entertainment applications [2] have been developed. These applications require specific and stringent QoS and security demands: very low end-to-end latency, which can be less than $1 \mathrm{~ms}$, a high transmission rate for huge data exchange. In addition, some use cases require ultra-reliability of $99.99 \%$ [11]. Therefore, different various Medium Access Control (MAC) [2] protocols have been proposed and developed in order to handle network access and transmission with minimum packet loss. While the CSMA-based IEEE $802.11 \mathrm{p}$ protocol is the algorithm currently proposed to share the radio medium between users, a number of criticisms have arisen, pointing out the drawbacks of this proposal. Today, thousands of cars are already equipped with IEEE $802.11 \mathrm{p}$ devices. Thus, any NGV device proposition to improve performance must retaining full coexistence and interoperability with the IEEE 802.11 p.

In the meantime, the community has investigated access protocols based on TDMA for access in VANETs, ( see, for instance, [2] and [14]).

Recently, a new TDMA-based protocol, called AS-DTMAC, that uses active signaling has been proposed. To the authors' best knowledge, using active signaling in a TDMA scheme leads to one of the first protocols to combine the advantages of random access (i.e. small access delay for sporadic traffic) and with the advantages of TDMA (stability of the access rights and hidden collision avoidance). AS-DTMAC, is detailed in [3], [4].

This paper aims to study the coexistence of the IEEE 802.11p protocol and AS-DTMAC and to compute two metrics related to this coexistence. The first one represents the probability that an IEEE 802.11 p packet will interfere in the selection process of AS-DTMAC, while the second one referes to the waiting time for an AS-DTMAC vehicle to be able to access to the channel. The remainder of the paper is organized as follows. Section II presents related work. Section III briefly describes the AS-DTMAC protocol. Section IV explains the coexistence between the IEEE 802.11p protocol and ASDTMAC and derives performance metrics related to this coexistence. Section V concludes this paper.

\section{RELATED WORK}

The Medium Access Control (MAC) layer has the role of sharing the radio channel by an efficient and reliable access to the channel for each node present in the network. In VANETs, the continuous and fast change in network topology due to high node mobility, makes the design of the MAC more difficult to handle [2]. To meet these requirements, the design of a new MAC layer is essential. Recently, several MAC protocols for VANETs have been proposed in the literature [12] [14] [15] [16], each of them treating a particular problem in a specific mobility scenario. As in traditional ad hoc networks, MAC protocols in VANETs belong to one of two broad categories: contention-based and contention-free.

In contention-based protocols, each node can try to access the channel when it has data to transmit, generally using the carrier sensing mechanism [7]. The emerging standard in VANETs: IEEE 802.11p [8] is a Contention-based MAC protocol which uses a priority-based access scheme that employs both Enhanced Distributed Channel Access (EDCA) and Carrier Sense Multiple Access with Collision Avoidance (CSMA/CA) mechanisms [9]. Contention-based MAC protocols can not provide a reliable broadcast mechanism with bounded communication delay, and thus handling safety messages is highly challenging for the IEEE 802.11p standard, which has to rely on the EDCA scheme to support timebounded traffic. But although EDCA improves the access of 
urgent packets, it does not correct all the problems of the underlying CSMA/CA access scheme.

In Contention-free MAC protocols, only one vehicle within a given neighborhood can access the channel at a given time and in a given area. Therefore, these protocols provide collision-free transmission with bounded access delay for realtime applications. In recent years, many distributed TDMAbased MAC protocols have been proposed to guarantee realtime and reliable communications in VANETs while avoiding the access and the merging collision problems. An access collision problem occurs when two or more vehicles within the same two-hop neighborhood set attempt to access the same available time slot, a problem which is likely to happen when a distributed scheme is used [2]. A merging collision problem occurs when two vehicles in different two-hop sets to access the same time slot become members of the same two-hop set due to changes in their position [6].

Each protocol has been proposed for a particular problem in a specific mobility scenario. For instance, the authors in [10] have proposed an ADHOC Medium Access Control protocol (ADHOC MAC) to provide an efficient broadcast service for inter-vehicle communications and solve MAC issues such as the hidden-exposed terminal problem and QoS provision. ADHOC MAC is a contention-free medium access protocol which implements a dynamic TDMA mechanism that is able to provide prompt access based on a distributed access technique, $\mathrm{R}$ ALOHA (Reliable R-ALOHA [13]). Each vehicle can access the channel at least once in each frame by randomly selecting a time slot as its Basic CHannel (BCH). In [14], Omar et al. developed and evaluated a contention-free multi-channel MAC protocol proposed for VANETs, called VeMAC. This protocol supports efficient one-hop and multi-hop broadcast services on the control channel without the hidden terminal problem caused by node mobility. These broadcast services are presented in [10] for ADHOC MAC. VeMAC reduces the collision rate by assigning disjoint sets of time slots to vehicles moving in opposite directions (Left, Right) and to Road Side Units (RSUs). ATSA [15] (medium Access Tdma Slot Assignement protocol) and DATS [16] (Decentralized Adaptive Tdma Scheduling strategy) are improvements of VeMAC while using the same principles. Like VeMAC, ATSA divides the frame into two sets of time slots, Left and Right. However in ATSA, when a vehicle accesses the network, it chooses a frame length and competes for one of the time slots available for its direction.

The contribution of this paper is to show that a Contentionbased MAC protocol, IEEE $802.11 \mathrm{p}$, can coexist with a Contention-free MAC protocol, AS-DTMAC, and to compute metrics when these two protocols are used in the same VANET. Moreover, we propose simple modifications to ASDTMAC to enable it to better coexist with the IEEE 802.11p protocol.

\section{THE DTMAC PROTOCOL WITH ACTIVE SIGNALING (AS-DTMAC)}

AS-DTMAC [3] is a distributed TDMA, which is mainly based on the DTMAC protocol [12]. The main idea of the DTMAC is to divide the road into different zones $\left(x_{i}, i=\right.$ $1, \ldots, N)$ according to the communication range of the vehicles, denoted by $R$. In this way, we can impose a new concept of slot reuse, which consists in reusing the slot spatially. As described in [12], the vehicles in zone $x_{i}$ can use the same set of slots as the vehicles in $x_{i+3}$. The only condition to this spatial reuse is that the distance between simultaneously transmitting vehicles must be greater than $2 \times R$. Furthermore, the slot scheduling table is updated each time a packet is sent by a vehicle. The packet sent contains a special field, named frame information, which specifies the status of slots. Thus, it will be easy for all vehicles to select the available slots in the next frame. DTMAC acts like a slotted Aloha protocol on the 'non-busy' slots of the frame. This protocol can be applied to highway scenarios and supports different parameters (vehicles moving in opposite directions, varying speed, varied traffic density). Thanks to GPS technology, each vehicle is able to have information about its position and the exact time. This information is useful for the functionality of DTMAC.

The new version of the protocol called AS-DTMAC aims to enhance the robustness of the background algorithm (DTMAC) against collisions by using the actif signaling mechanism. Figure 1 illustrates the inclusion of this mechanism in the slot. During the signaling part of the packet, a selection process is carried out to obtain exactly one packet to be sent in the payload part of the slot.

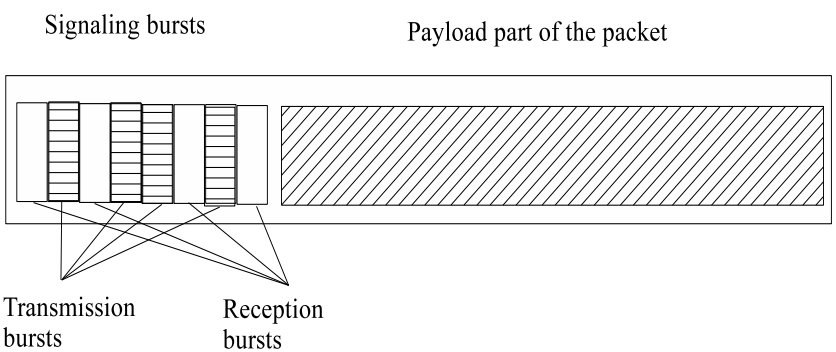

Figure 1. Slot structure of the active signaling mechanism

The active signaling part of the slot consists of $n$ mini-slots, each of which could be a transmission or a listening period. This succession is dictated by a randomly generated binary key. ' 1 ' means that the vehicle with a packet to send transmits during the signaling bursts. ' 0 ' means that the vehicle with a packet to send senses the channel during this mini-slot. When a vehicle selects a listening period and senses a transmission, the competition to get the slot is over. For instance, a vehicle that draws the key '01001110' will listen during the first mini-slot and if no competing transmission is sensed during this minislot, it will transmit during the next mini-slot. The following two steps in the selection process will be two listening periods. 
The selection process continues using the same rule until the key is completely used up.

In the description above, $n$ selection mini-slots are used in the selection process of the actif signaling. In most cases we will assume that the selection key which encompasses $n$ bits is selected at random. If a vehicle has an emergency message to transmit, these keys could be handled using other techniques, in particular we can create priorities. In this case, the vehicles that require immediate access will set the first bit to '1'. Thus, these vehicles will have a guaranteed priority access over the set of vehicles that are trying to get a slot using the standard scheme. These vehicles will keep the first bit set to ' 0 '.

\section{COEXISTEnCE}

We start by studying the general behavior of the two protocols when they coexist. That is the focus of Section IV-A. In Section IV-B we determine the possible interaction in the AS-DTMAC selection process. Then we study the waiting time of AS-DTMAC after the beginning of an IEEE 802.11p transmission. This task is the subject of Section IV-C.

\section{A. Behavior of the two protocols in coexistence mode}

The IEEE $802.11 \mathrm{p}$ protocol is a CSMA/CA carrier sensebased protocol and thus coexistence with another protocol is possible. When an IEEE $802.11 \mathrm{p}$ vehicle senses a busy channel, it waits for the end of the transmission and then, after a given idle time interval, it sends its packet. If ASDTMAC packets are sent in the channel, the IEEE 802.11p vehicles will wait for an empty slot to start their transmissions. Thus, on the one hand the IEEE 802.11p can coexist with the transmission of AS-DTMAC packets. On the other hand, if an AS-DTMAC transmission is started on a given slot, the selection procedure will continue even if IEEE 802.11p vehicles try to send packets, as we will show more precisely in Subsection IV-B below. In fact, the interference of an IEEE 802.11p transmission in the AS-DTMAC selection process is very unlikely. Equally importantly, AS-DTMAC will not affect the IEEE 802.11p transmission. With active signaling, ASDTMAC will rapidly detect an IEEE 802.11 p transmission during the sensing interval of the selection phase. Thus the interference produced by AS-DTMAC will be of short duration and the IEEE 802.11 p transmission is likely to remain successful even if there is some interference by short transmission bursts.

When a slot initially reserved by a vehicle using ASDTMAC is used by another vehicle (thus in principle a vehicle using IEEE 802.11p) we propose to use AS-DTMAC on the coming slot of the synchronous frame using a greedy approach and to maintain the slot reserved on the next frame of the ASTDMAC protocol. If the load of the IEEE 802.11p vehicles is not too high, there is a high probability that the AS-TDMAC vehicle will recover its slot on the next frame of AS-TDMAC.

If we suppose that the load of the IEEE 802.11p vehicles is high then the reservation protocol of AS-TDMAC can be seriously affected by the IEEE 802.11 p traffic. In this case we propose to use AS-TDMAC in a fully asynchronous mode.
The protocol will operate completely asynchronously. At the arrival of one packet in a vehicle, the radio modem will sense the channel. If the channel is idle the packet is sent with the active signaling sent as a preamble to resolve any possible collision. If the channel is busy, the node senses the channel until it is free and sends the packet, also sending the active signaling bursts before the transmission. At high load, we observe that the AS-DTMAC protocol preempts the channel to IEEE 802.11. This can be explained by the generalized CMSA scheme embedded in AS-DTMAC which is a more efficient access scheme than the simple CSMA backoff technique of IEEE 802.11p.

\section{B. Interaction of IEEE 802.11 in the AS-DTMAC selection process}

The model we assume is that an AS-DTMAC node starts its transmission at the beginning of a slot. We assume that at the beginning of the slot there is no IEEE 802.11p transmission present. The IEEE 802.11 p nodes can interfere with the ASDTMAC transmission if an IEEE 802.11p packet can start during the listening period of the selection phase of the AS-DTMAC transmission. We know that the IEEE 802.11p protocol starts sending a packet after sensing that the channel has been continuously idle for an inter-frame period (DIFS) plus possibly the backoff time.

To fix the idea, we use the following values DIFS $=58 \mu \mathrm{s}$ and we assume that the mini-slot time of the selection process of AS-DTMAC is of the same duration as a short inter-frame in IEEE $802.11 \mathrm{p}$ (SIFS), thus this duration is $13 \mu \mathrm{s}$. A duration greater than or equal to DIFS will exist in the AS-DTMAC selection process if a listening interval of at least $a=\left\lceil\frac{D I F S}{S I F S}\right\rceil$ consecutive SIFSs occurs in the AS-DTMAC selection bursts. This is a necessary condition for IEEE 802.11p to interfere with an AS-DTMAC transmission ${ }^{1}$. We suppose that $n$ is the number of AS-DTMAC contending vehicles. The probability $P_{r}$ that an IEEE $802.11 \mathrm{p}$ node can interfere in the AS-DTMAC selection process is thus

$$
P_{r}=1 / 2^{n a} .
$$

This probability $P_{r}$ is given below for $\left\lceil\frac{D I F S}{S I F S}\right\rceil=5$ which corresponds to the figures we has chosen:

\begin{tabular}{|c|c|c|c|c|}
\hline$n$ & 1 & 2 & 3 & 4 \\
\hline$P_{r}$ & $3.1 e^{-2}$ & $9.7 e^{-4}$ & $3.0 e^{-5}$ & $9.5 e^{-7}$ \\
\hline
\end{tabular}

We observe that this probability is very small, thus the IEEE $802.11 \mathrm{p}$ protocol is very unlikely to interfere in the ASDTMAC selection process if the IEEE 802.11p transmission has not started previously, even if an AS-DTMAC stable mode is established where most of the nodes already have a reserved slot in the AS-DTMAC frame. In this condition we have $n=1$. Moreover in the previous computation we ignored the backoff time of IEEE 802.11p, thus the probability of an IEEE 802.11p packet interfering with AS-DTMAC is less than $3.1 e^{-2}$.

\footnotetext{
${ }^{1}$ We omit the back off time of IEEE $802.11 \mathrm{p}$
} 
In the next section we study the waiting time of ASDTMAC if an IEEE 802.11p transmission has started.

\section{Waiting time for AS-DTMAC after the beginning of an IEEE 802.11p transmission}

We adopt the following simplified model. The IEEE 802.11p transmission traffic is a Poisson distribution of rate $\lambda$. We suppose that the duration of an IEEE 802.11p packet is one unit and we include in this duration the packet itself and the duration of the MAC overhead: inter-frame and possible back-off. If the IEEE 802.11 p activity is such that the interframe between the IEEE 802.11p does not coincide with the beginning of the AS-DTMAC slots, the IEEE 802.11p will block the access of the AS-DTMAC packets. We will assume that to be the case in the following development where we evaluate a busy period of IEEE 802.11 p packets.

To carry out such a task, we use the M/D/1 queue model. With our assumptions, the duration of the busy period of IEEE 802.11p packets is exactly the duration of the busy period of the M/D/1 queue. It is possible to compute the Laplace transform $\beta(s)$ of the density of the busy period of the M/D/1 queue noted $f(t)$.

For $s$ such that $\Re(s) \geq 0, \beta(s)$ is equal to the root with the smallest absolute value in $z$ of the equation:

$$
z=\exp (-s-\lambda(1-z))
$$

We can introduce the Lambert function which is denoted by $W(\cdot)$ and we have $z=w e^{w} \Longleftrightarrow w=W(z)$. With this function we can easily show that the Laplace transform of the busy period is:

$$
\beta(s)=-\frac{1}{\lambda} W\left(-\lambda e^{-\lambda-s}\right) .
$$

If we note by $X$ the duration of the busy period for the M/D/1 queue, and $g(x)=P b(X>x)=\int_{x}^{\infty} f(t) d t$ ( $x$ is the busy period in time units, in other word the waiting time for the AS-DTMAC packet), the Laplace transform of $g(x)$ is:

$$
G(s)=\frac{1-F(s)}{s}=\frac{1-\beta(s)}{s} .
$$

Since it is not possible to recognize in $G(s)$ the Laplace transform of some known functions, we have to rely on numerical techniques. We use the well-known inverse formula named the Bromwich-Mellin transform and we have:

$F(p)=\int_{0}^{\infty} e^{-p t} f(t) d t \Longleftrightarrow f(t)=\frac{1}{2 i \pi} \int_{c-i \infty}^{c+i \infty} e^{t s} F(s) d s$

where $c$ is a real number such that all the singularities of $F(s)$ have a real part that is smaller than $c$. We can show using the prevision formula and [5] that for $A>0$ :

$$
\begin{aligned}
g(t) & =\frac{e^{A / 2}}{2 t} \sum_{k=\infty}^{k=\infty}(-1)^{k} \Re\left(G\left(\frac{A+2 k \pi i}{2 t}\right)\right) \\
& -\sum_{k=1}^{k=\infty} e^{-k A} g((2 k+1) t) .
\end{aligned}
$$

Since $|g(t)|<1$, the last term of the previous equation, is bounded by:

$$
\frac{e^{-A}}{1-e^{-A}} \simeq e^{-A}
$$

Thus $g(t)$ can be approximated by $g_{n}(t)$ with :

$$
\begin{aligned}
g_{n}(t) & =\frac{e^{A / 2}}{2 t} \Re\left(G\left(\frac{A}{2 t}\right)\right) \\
+ & \frac{e^{A / 2}}{t} \sum_{k=1}^{k=n}(-1)^{k} \Re\left(G\left(\frac{A+2 k \pi i}{2 t}\right)\right)
\end{aligned}
$$

The sequence $g_{n}(t)$ is usually an alternate sequence and so it is useful to consider an acceleration technique. It is shown in [5] that the Euler summation is generally a good choice. Thus we use the following approximation:

$$
g(t) \simeq \sum_{k=0}^{m}\left(\begin{array}{c}
m \\
k
\end{array}\right) 2^{-m} g_{n+m}(t)
$$

In our numerical result we use $A=18, n=11$ and $m=10$ as in [5].

In Figure 2, we show the distribution function of the busy period for $\lambda=0.2$. This busy period corresponds to the waiting time for an AS-DTMAC packet which waits to access when IEEE 802.11p packets are sent. As expected, we observe a step function that comes as a result of the transmission time of 1 unit (u). In Figures 3, 4 and 5 we show the distribution functions of the busy period for $\lambda=0.4,0.6$ and $\lambda=0.8$, respectively. We look for the smallest duration $T$ for which the probability is less than 0.05 that the waiting time for a free channel exceeds $T$. In other words, $T$ will give for ASDTMAC the maximum access delay to the channel with a priority greater than 0.95 when a Poisson traffic of load $\lambda$ is using the IEEE $802.11 \mathrm{p}$ access protocol. For $\lambda=0.2$ we obtain $T=3$, for $\lambda=0.4$ we have $T=5$, for $\lambda=0.6$ we have $T=9$ and for $\lambda=0.8$ we have $T=21$. We observe that except for a high load $\lambda=0.8$, the maximum waiting time remains small. If we need to reduce this delay, we can adapt AS-DTMAC. Instead of using a synchronous access, we can use AS-DTMAC in an asynchronous mode. In that case, ASDTMAC will preempt the channel, as shown by the evaluation in Subsection IV-B.

In Figure 6, we show the average waiting time for ASDTMAC packet versus the channel load. This result is deduced from the distribution function of the busy period for each $\lambda$. As we can see this delay remains resonable up to load $\lambda=$ 0.8 which shows the good performances of the AS-DTMAC protocol. 


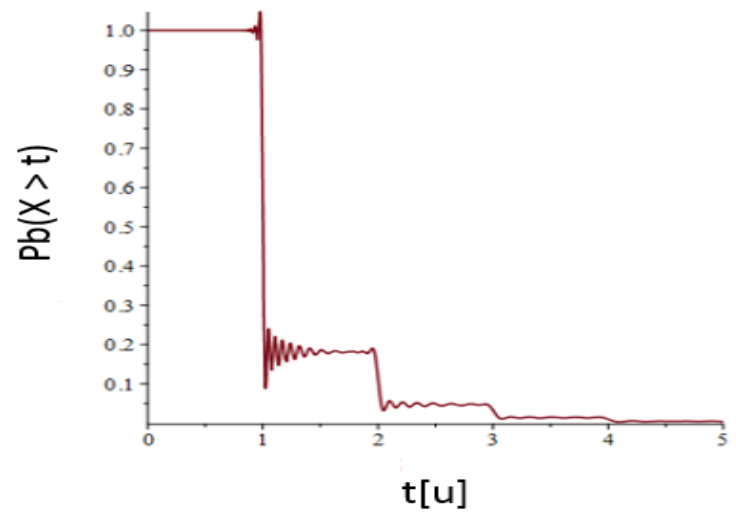

Figure 2. Distribution function of the busy period $\mathrm{Pb}(X>t)$ for $\lambda=0.2$ $t$ is in time unit $(\mathrm{u})$

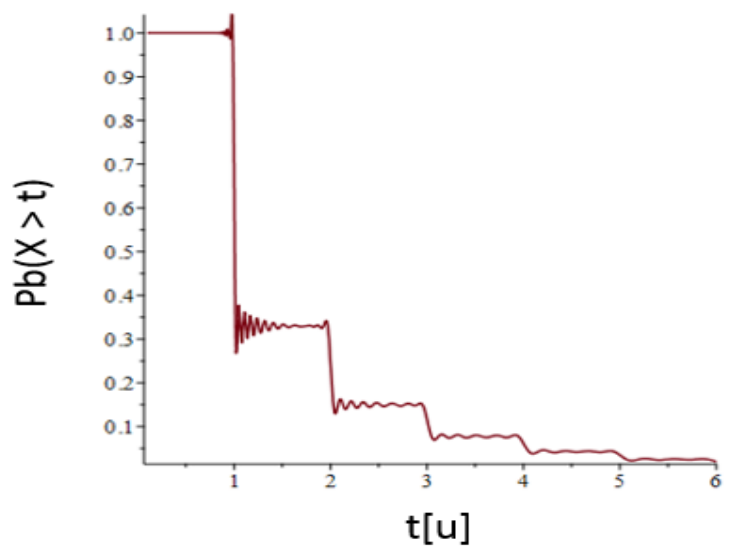

Figure 3. Distribution of the busy period $\operatorname{Pb}(X>t)$ for $\lambda=0.4, t$ is in time unit (u).

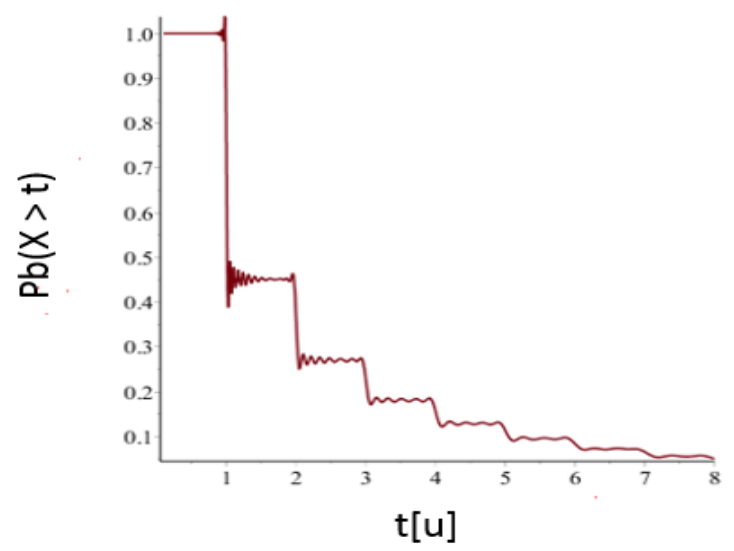

Figure 4. Distribution of the busy period $\mathrm{Pb}(X>t)$ for $\lambda=0.6, t$ is in time unit (u).

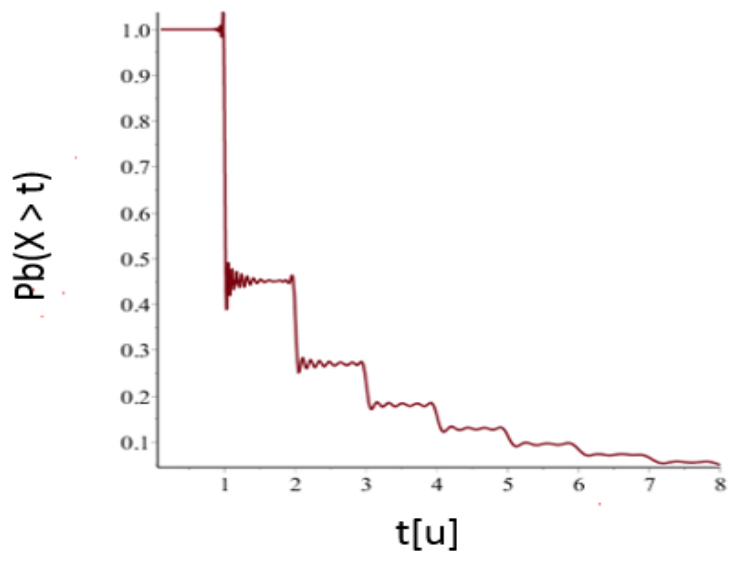

Figure 5. Distribution of the busy period $\operatorname{Pb}(X>t)$ for $\lambda=0.8, t$ is in time unit $(\mathrm{u})$.

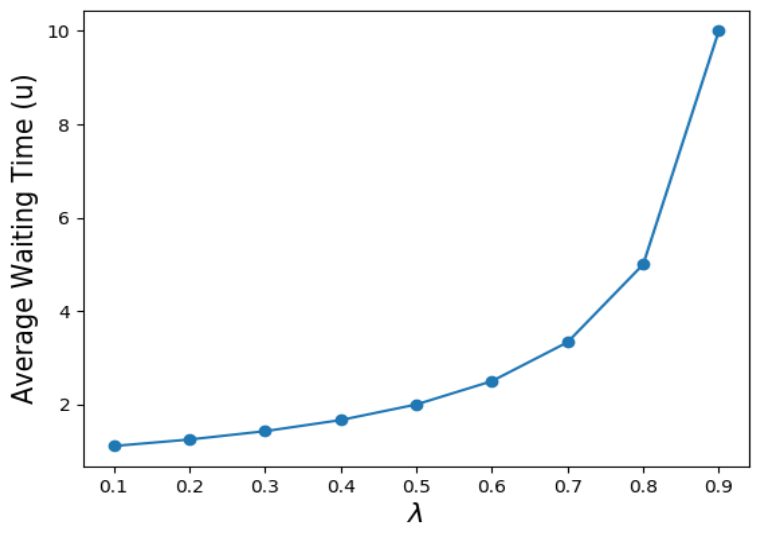

Figure 6. Average waiting time for an AS-DTMAC packet

\section{CONCLUSion}

We have shown that the contention-based IEEE 802.11p protocol can coexist with the contention-free AS-DTMAC protocol. We have studied the probability that an IEEE $802.11 \mathrm{p}$ transmission can interfere with the AS-DTMAC selection process and to preempt an AS-DTMAC transmission. This probability is very small, indicating, on the one hand, that an AS-DTMAC transmission is very unlikely to be preempted by an IEEE $802.11 \mathrm{p}$ transmission. On the other hand, when IEEE $802.11 \mathrm{p}$ transmissions are established, the AS-DTMAC, being a TDMA system, can not in principle preempt the transmission. We have studied the distribution of the waiting time for an AS-DTMAC user to be able to access to the channel. We have shown that, if needed, AS-DTMAC can possibly preempt the flow of IEEE 802.11p transmissions if AS-DTMAC operates asynchronously. In this case, the ASDTMAC user has only to transmit just after the IEEE 802.11p transmission. If we use this operating mode, AS-DTMAC has priority over IEEE $802.11 \mathrm{p}$ transmissions. Moreover, the first 
bit of the transmission key in AS-DTMAC allows one to prioritize access for AS-DTMAC users.

A fairness index showing the ability of IEEE $802.11 p$ devices to have the same opportunities as NGV devices to access the channel will be the subject of another contribution. Also, the model assumes a perfectly symmetric transmission (reception) range situation. Therefore, it would be interesting to show how an asymmetric scenario would affect the coexistence of the two protocols. Moreover, we plan to continue to investigate the other specifications required for the design of the IEEE 802.11bd, such as the ability of NGV devices to run in a mode in which they can interoperate with IEEE 802.11p devices, a property that is defined in the literature such as the Backward compatibility.

\section{REFERENCES}

[1] Fei Hu, Security and Privacy in Internet of Things (IoTs): Models Algorithms, and Implementations, ISBN 978-1-4987-2318-3.

[2] M. Hadded, P. Muhlethaler, A. Laouiti, R. Zagrouba, and L. A. Saidane, TDMA-based MAC protocols for vehicular ad hoc networks a survey, qualitative analysis and open research issues, IEEE Communications Surveys Tutorials, vol. 17, no. 4, pp. 2461-2492, Jun. 2015.

[3] F. Boukhalfa, M. Hadded, P. Muhlethaler and O. Shagdar, An active signaling mechanism to reduce access collisions in a distributed TDMA based MAC protocol for vehicular networks" Advanced Information Networking and Applications, AINA-2019, Kunibiki Messe, Matsue, Japan March 27 to March 29, 2019.

[4] F. Boukhalfa, M. Hadded, P. Muhlethaler, and O. Shagdar, An Analytical Model for Performance Analysis of an Active Signaling-based TDMA MAC Protocol for Vehicular Networks, In Proceedings of the 2019 IEEE 90th Vehicular Technology Conference (VTC2019-Fall), Honolulu, HI, USA, Sep. 2019.

[5] J. Abate and W. Whitt, Numerical Inversion of Laplace Transforms of Probability Distributions, ORSA Journal on Computing, Vol 7, no 1, pp 38-43, 1995.
[6] M. Hadded, R. Zagrouba, A. Laouiti, P. Muhlethaler, and L. A. Saidane, An optimal strategy for collision-free slots allocations in vehicular adhoc networks, in Advances in Intelligent Systems and Computing, vol. 306, Kuala Lumpur, Malaysia, Jun. 2015, pp. 1530.

[7] F. Ye, R. Yim, J. Zhang, and S. Roy, Congestion control to achieve optimal broadcast efciency in vanets, in IEEE International Conference on Communications (ICC), Cape Town, South Africa, May 2010, pp. 1-5.

[8] $802.11 p-2010$, IEEE standard for information technology - Telecommunications and information exchange between systems - local and metropolitan area networks - specific requirements part 11 : Wireless LAN medium access control (MAC) and physical layer (PHY) and physical layer (PHY) specifications amendment 6: Wireless access in vehicular environments Std., 2010.

[9] R. Uzcategui and G. Acosta-Marum, Wave: A tutorial, IEEE Communications Magazine, vol. 47, no. 5, pp. 126-133, May 2009

[10] F. Borgonovo, A. Capone, M. Cesana, and L. Fratta, Adhoc mac: new mac architecture for ad hoc networks providing efficient and reliable point-to-point and broadcast services, Wireless Networks, vol. 10, no. 4, pp. 359-366, 2004.

[11] G. Naik,B. Choudhury and J. M Park, IEEE 802.11 bd \& $5 G N R$ V2X Evolution of Radio Access Technologies for V2X Communications, IEEE Access journal, vol. 7, pp. 70169-70184, May. 2019.

[12] M. Hadded, A. Laouiti, P. Muhlethaler, and L. A Saidane, An infrastructure-free slot assignment algorithm for reliable broadcast of periodic messages in vehicular ad hoc networks, in Vehicular Technology Conference VTC-Fall, Montreal, Canada, Sep. 2016.

[13] F. Borgonovo, A. Capone, M. Cesana, and L. Fratta, Rr-aloha, a reliable r-aloha broadcast channel for ad-hoc intervehicle communication networks, in IEEE IFIP Annual Mediterranean Ad Hoc Networking Workshop (Med-Hoc-Net), Baia Chia, Italy, 2002.

[14] W. Zhuang, H. A. Omar, and L. Lio, Vemac: A novel multichannel mac protocol for vehicular ad hoc networks, in IEEE Conference on Computer Communications Workshops (INFOCOM WKSHPS), Shanghai, China, Aug. 2011, pp. 413-418.

[15] Y. Weidong, L. Pan, L. Yan, and Z. Hongsong, Adaptive TDMA slot assignment protocol for vehicular ad-hoc networks, Journal of China Universities of Posts and Telecommunications, vol. 20, no. 1, pp. 1118, Feb. 2013.

[16] W. Ke, Y. Weidong, L. Pan, and Z. Hongsong, A decentralized adaptive TDMA scheduling strategy for VANETs, in IEEE Wireless Communications and Networking Conference Workshops (WCNCW), Shanghai, China, Apr. 2013, pp. 216221. 